\title{
Hepatocellular-Cholangiocarcinomas in Non-alcoholic Fatty Liver Disease: Evidence for Stem Cell Origin
}

\author{
Paulo Giovanni Mendoza, ${ }^{1}$ Wilson Tsui, ${ }^{2}$ Lorraine Ho,${ }^{3}$ Michael Wayne, ${ }^{4}$ Alexander Kagen, ${ }^{5}$ Neil Theise ${ }^{6}$
}

${ }^{1}$ Department of Pathology and Laboratory Medicine, National Kidney \& Transplant Institute, East Avenue, Quezon City, Philippines ${ }^{2}$ Department of Pathology, Caritas Medical Centre, Hong Kong, China

${ }^{3}$ Department of Radiology, Caritas Medical Centre, Hong Kong, China

${ }^{4}$ Department of Surgery, Beth Israel Medical Center of Albert Einstein College of Medicine, New York, New York, USA

${ }^{5}$ Department of Radiology, Icahn School of Medicine at Mount Sinai, Mount Sinai West, Mount Sinai St. Luke's, New York, USA

${ }^{6}$ Departments of Pathology and Medicine (Division of Digestive Diseases), Beth Israel Medical Center of Albert Einstein College of Medicine,

New York, New York, USA

\section{ABSTRACT}

The authors present two cases of combined hepatocellular-cholangiocarcinoma in a background of noncirrhotic, non-alcoholic fatty liver disease (NAFLD). The increasing incidence of NAFLD and the subsequent recognition of it being a pre-malignant condition even in the absence of significant fibrosis or established cirrhosis has led to the investigations of the different pathways involved in NAFLD-associated hepatocarcinogenesis, including speculations regarding the possibility that many derive from pre-malignant hepatocellular adenomas, tumors also increasingly associated with NAFLD, or reflect malignant transformation of mature hepatocytes through genetic and epigenetic alterations reflecting inflammatory changes in NAFLD. However, NAFLD, like most chronic liver diseases, leads to progressive activation of resident hepatobiliary stem/progenitor cells that are thought to give rise to malignant tumors in other settings. In particular, combined hepatocellularcholangiocarcinomas (with and without stem cell features) are thought to reflect malignant transformation of these activated progenitors. Our two cases of combined hepatocellular-cholangiocarcinomas suggest that malignant transformation of hepatobiliary stem/progenitor cells in NAFLD are also a possible pathway to malignancy, even in the absence of established cirrhosis.

Key words: hepatocellular-cholangiocarcinoma, non-alcoholic fatty liver disease (NAFLD), steatohepatitis, stem cell

\section{ISSN 0118-3265}

Printed in the Philippines.

Copyright $(92016$ by the PJP.

Received: 4 April 2016.

Accepted: 2 May 2016.

Published online first: 5 May 2016.

http://dx.doi.org/10.21141/PJP.2016.008

Corresponding author: Paulo Giovanni L. Mendoza, MD Email: pgmendozamd@hotmail.com

\section{INTRODUCTION}

Non-alcoholic fatty liver disease (NAFLD) is now the most common form of chronic liver disease in industrialized countries. ${ }^{1}$ The histologic changes seen in patient livers include steatosis, steatohepatitis, and steatofibrosis. Many patients progress to cirrhosis and its associated complications of liver failure and hepatocellular carcinoma (HCC). Studies indicate that development of HCC in cirrhotic patients with NAFLD has a yearly incidence of 2\%-5\%. ${ }^{2}$ Recently, reports of HCC developing in NAFLD in the absence of cirrhosis have started to surface. ${ }^{3-4}$ Currently, individuals who have NAFLD without underlying cirrhosis are not screened routinely for HCC at most centers because of the assumed low risk of cancer development, though such cases of pre-cirrhotic HCC raise questions in this regard.

Hypotheses about the pathogenesis of non-cirrhotic, NAFLDassociated HCC have been suggested. One proposal suggests that these cases developed from malignant transformation of hepatocellular adenoma (HCA), particularly the telangiectatic subtype and those with $\beta$-catenin mutation, though whether HCC and HCA develop simultaneously or successively has not been clearly resolved. ${ }^{2,5}$ Some studies suggest that tumor suppressor genes such as phosphatase and tensin homolog (PTEN), promyelocytic leukemia and p53 play an important role in the development of steatosis with associated liver cell damage. ${ }^{6-7}$ It follows that the loss of tumor suppression could promote formation of HCC, even 
without cirrhosis. Another mechanism that may contribute is the dysregulation of bile acid metabolism, which may induce hepatocyte apoptosis promoting $\mathrm{HCC}$ in the setting of steatosis. ${ }^{8}$ These various hypotheses all generally point toward tumor development from preexisting hepatocytes that undergo malignant transformation.

We present two cases of combined hepatocellularcholangiocarcinoma (HCC-ChC) developing in a setting of NAFLD, one without significant scarring and the other with "incomplete septal cirrhosis" (possibly regressed fibrosis). The combination of histomorphology and immunostaining (stains summarized in Table 1) confirmed the tumor diagnoses. The presence of mixed hepatobiliary tumors, one containing an overt stem cell variant, raises the possibility that some hepatobiliary malignancies in non-cirrhotic NAFLD arise through the activation and malignant transformation of pluripotent liver stem/progenitor cells.

\begin{tabular}{|c|c|c|c|}
\hline Antibody & Differentiation & Company & Titer \\
\hline Hep Par 1 & Hepatocytic lineages & Dako & $1: 400$ \\
\hline Keratin 19 & $\begin{array}{l}\text { Cholangiocytic and Stem / } \\
\text { Progenitor cell lineages }\end{array}$ & Dako & $1: 100$ \\
\hline EрCAM & $\begin{array}{l}\text { Cholangiocytic and Stem / } \\
\text { Progenitor cell lineages }\end{array}$ & Leica & $1: 250$ \\
\hline CD56 & $\begin{array}{l}\text { Stem / Progenitor cell } \\
\text { lineages, Neuroendocrine } \\
\text { differentiation }\end{array}$ & Leica & $1: 250$ \\
\hline Arginase-1 & Hepatocytic lineages & Sigma & $1: 8000$ \\
\hline Keratin 7 & $\begin{array}{l}\text { Cholangiocytic and Stem / } \\
\text { Progenitor cell lineages }\end{array}$ & Dako & $1: 1000$ \\
\hline Canalicular CD10 & Hepatocytic lineages & Leica & $1: 250$ \\
\hline $\begin{array}{l}\text { Canalicular CEA } \\
\text { (polyclonal) }\end{array}$ & Hepatocytic lineages & Dako & $1: 6000$ \\
\hline
\end{tabular}

\section{CASE 1}

A 49-year-old-woman, from the island of St. Vincent, but residing in New York City, was referred to Hepatology clinic for serum alkaline phosphatase elevation and sonogram with fatty liver. She denied any symptoms of fatigue, weight loss, pruritis, abdominal pain or distention. Past medical history revealed hypertension, dyslipidemia and pre-diabetes. Family history was significant for sarcoidosis. On physical exam, she was obese (body mass index of $31.0 \mathrm{~kg} / \mathrm{m}^{2}$ ), had peri-orbital swelling and was in no acute distress. She had no stigmata of chronic liver disease or hepatosplenomegaly.

Pertinent laboratory tests included serum alkaline phosphatase (ALP) of 223 U/L (normal: 38-126), serum aspartate aminotransferase (AST) $37 \mathrm{U} / \mathrm{L}$ (normal: 15-46), serum alanine aminotransferase (ALT) 66 U/L (normal: 13-69), GGT 275 U/L (normal: 0-51) and angiotensin converting enzyme (ACE) $85 \mathrm{U} / \mathrm{L}$ (nl: 8-57). Total cholesterol $>240 \mathrm{mg} / \mathrm{dl}$ (normal: $<200 \mathrm{mg} / \mathrm{dl}$ ) and low density lipoprotein $(\mathrm{LDL})>160 \mathrm{mg} / \mathrm{dl} \quad($ normal: $<100)$ were significantly elevated. Serologic studies for hepatotropic viral infection and autoimmune disease were negative. Ceruloplasmin and alpha-1antitrypsin levels were within normal range. A computed tomography (CT) of the abdomen revealed fatty infiltration of the liver with no demonstration of hepatic mass. Ultra-sound guided liver biopsy was performed to evaluate the patient's chronic liver disease. Following confirmation of NAFLD (and exclusion of sarcoidosis), she was referred to a nutritionist and recommended to lose weight.

For approximately two years thereafter, the patient had close follow up with her primary care physician in Beth Israel Medical Center's Hepatology Clinic. Regularly scheduled blood work showed persistent elevation of her ALP and ALT levels with the range of 89-340 U/L and 66-289 U/L, respectively. She remained asymptomatic. After more than 2 year interval, a follow up CT scan of the abdomen and pelvis revealed a large, peripherally enhancing, hypodense lesion replacing much of the posterior segment of the right lobe of the liver with intrahepatic infiltration of the segment to the level of the porta hepatis. An MRI soon after confirmed the hepatic mass measuring $4.8 \times 5.2 \times 6.6 \mathrm{~cm}$.

Patient underwent a right hepatic lobectomy.

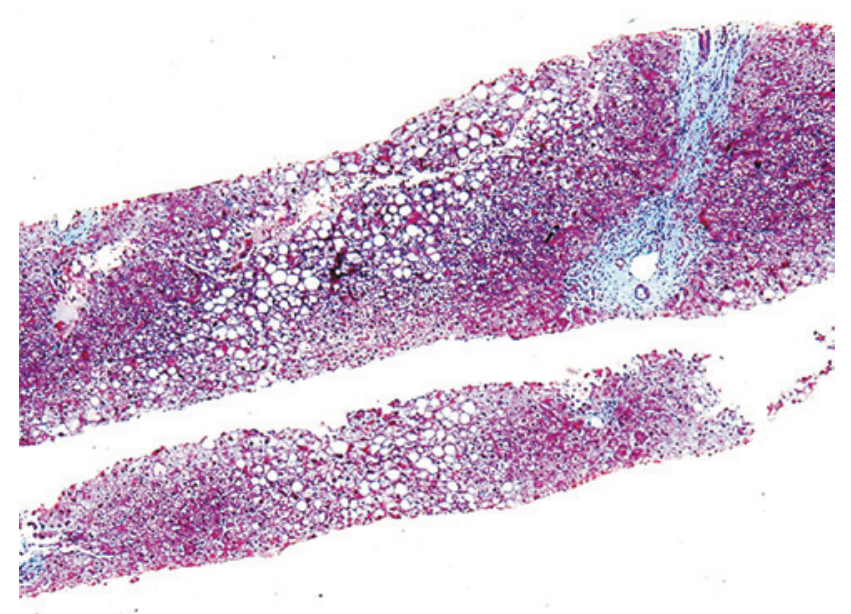

Figure 1. Liver tissue cores with steatosis and absence of pericellular fibrosis, (Trichrome stain, 20x, H\&E).

\section{Histopathology}

Needle biopsy

Multiple cores of liver tissue were submitted for evaluation. All of the cores showed a moderate degree of steatosis (Figure 1). Histologic steatohepatitis (i.e. hepatocyte ballooning with or without Mallory-Denk bodies, neutrophilic infiltration) was not present. Trichrome stain showed mildly increased portal stroma, but no features more specific for steatofibrosis. Hemosiderosis was absent with Prussian blue stain. Periodic acid-Schiff stain following diastase digestion showed no alpha-1-antitrypsin globules. Prominent ductular reactions, large cell change, and small cell change were not identified.

\section{Tumor resection}

The specimen was a partial hepatectomy of the right lobe. Grossly, the tumor was pale brown to cream in color and fibrotic in consistency. It measured $7 \mathrm{~cm}$ in greatest dimension, was irregular in shape and had ill-defined borders.

The histologic appearance of the non-tumoral liver showed an absence of the steatosis present in the prior biopsy specimen. Trichrome stain confirmed the mildly increased portal tract stroma, but again showed no steatofibrosis. Prominent ductular reactions, large cell change, and small cell change were not identified.

The histology of the mass is predominantly composed of malignant glands admixed with nests of tumor cells exhibiting hepatocellular morphologies (Figure 2). The glandular elements show a spectrum of moderate to poorly differentiated regions with some islands floating in mucin. Significantly, the nests of tumor cells with hepatocellular differentiation, have focal, peripheral small cells with increased nucleus:cytoplasmic ratio and nuclear hyperchromasia as have been identified in so-called "combined HCC-ChC with stem cell features, typical subtype” (Figure 2A) 


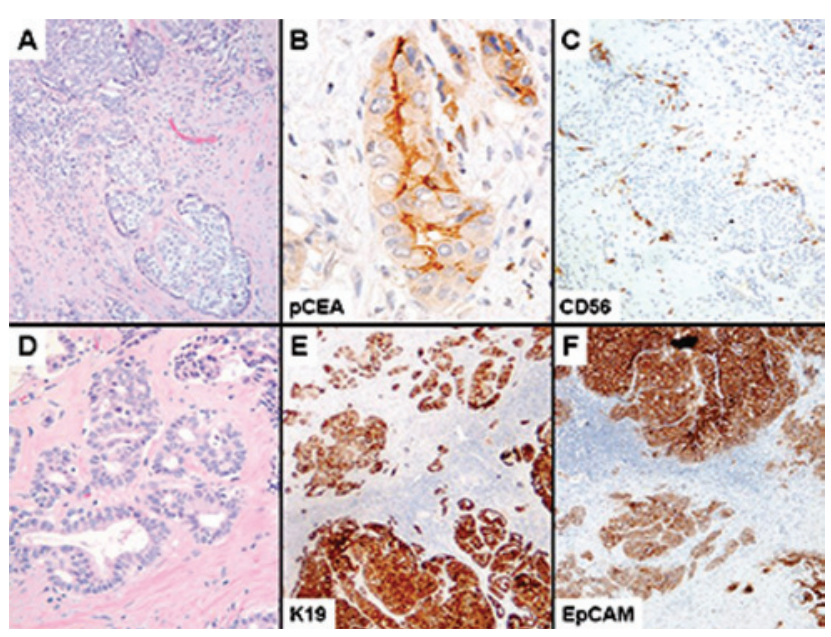

Figure 2. Case 1 tumor morphology (A) hepatocellular carcinoma admixed with (D) cholangiocarcinoma; (B) Positive canalicular staining with polyclonal CEA in HCC; (E) Keratin 19 with diffuse cytoplasmic staining in cholangiocarcinoma; (C) CD56 and (F) EpCAM highlight the stem/progenitor cell differentiation.

\section{Immunohistochemistry}

\section{Needle biopsy}

Immunostains for K19 and EpCAM did not demonstrate prominent ductular reactions or EpCAM positive hepatocytes.

\section{Tumor resection}

By immunohistochemistry, the areas with hepatocellular differentiation exhibited canalicular staining with polyclonal anti-CEA antibodies, but were negative for CD10, HepPar1 and arginase-1. Diffuse strong staining for keratins 7 and 19 and EpCAM were present throughout all forms of the tumor. The glandular components also showed cytoplasmic and luminal staining with polyclonal anti-CEA antibodies. The cuboidal cells surrounding the hepatocyte-like nests stain positively for CD56, a typical finding of the "typical" form of mixed hepatobiliary stem cell tumors. ${ }^{9,10}$

\section{CASE 2}

A 63-year-old male from Hong Kong presented in the clinic because of dizziness and vomiting. He is a known diabetic and hypertensive who presented with left sided weakness a few months prior to the present admission. On physical examination, he was conscious and alert with a blood pressure of $160 / 90 \mathrm{mmHg}$. Neither nystagmus nor other signs of cerebellar dysfunction were recognized. The lungs were clear and the abdomen was non-tender. There was no hepatomegaly or stigmata of chronic liver disease. His body mass index (BMI) was $23.9 \mathrm{~kg} / \mathrm{m}^{2}$. Laboratory tests showed elevated blood urea nitrogen, creatinine, glucose and triglycerides. Serum liver enzymes were not elevated nor were there serologic markers of hepatotropic viral infection or of autoimmune disease.

Upon sonographic assessment of the kidneys, an incidental, enhancing liver mass was found. The mass was seen next to the diaphragm, stomach and heart and was thus deemed technically too difficult to proceed with needle biopsy. The presence of a $2 \mathrm{~cm}$ mass with smooth outline was also confirmed via CT scan, revealing its close proximity to the surface and the risk of biopsy-associated rupture. Segmental resection of the bulging mass at segment 2 was done instead.
After the uneventful operation, patient was compliant with follow up visits and medications until three years when diabetic nephropathy worsened. He underwent dialysis treatment and suffered from another event of stroke. At this time, he started to complain of numbness, back pain and dysuria. Work up showed metastatic tumor foci, compressing the vertebra at T4 and T5 level.

\section{Histopathology}

The submitted specimen is a segmental resection with a bulging tumor on the surface. The $4 \mathrm{~cm}$ tumor was pale brown with alternating areas of hemorrhage. No necrotic or cirrhotic regions identified.

The non-tumoral liver parenchyma contained within the resection specimen shows incomplete septal cirrhosis (probably regressed cirrhosis) with features indicative of diabetes associated, nonalcoholic fatty liver disease, namely central-portal fibrous septa (Figure 3). Residual steatosis and histologic features of steatohepatitis are not identified. Prominent ductular reactions, large cell change, and small cell change were not present.

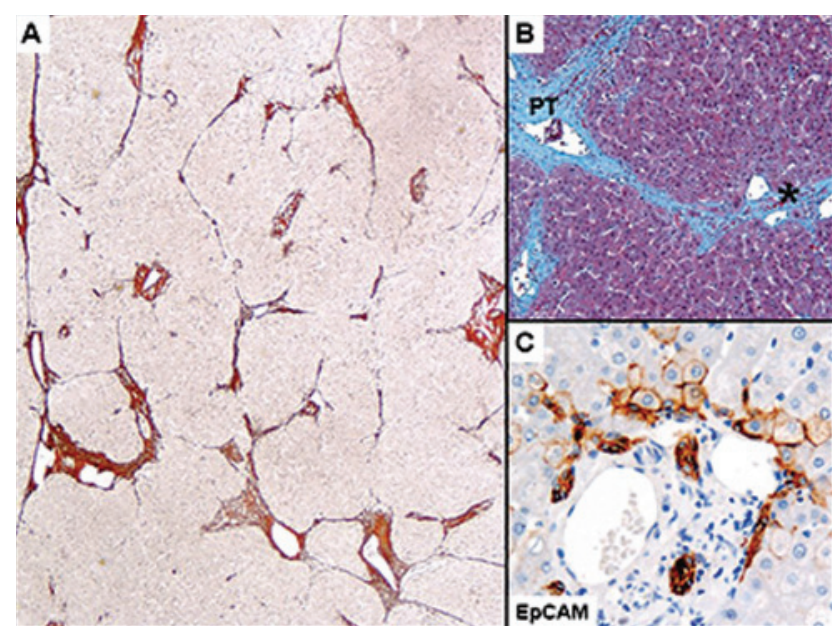

Figure 3. (A) Non-tumoral liver in case 2 with incomplete septal cirrhosis; (B) Trichrome stain highlighting septal fibrosis; (C) Positive membrane staining with EpCAM supporting stem cell features.

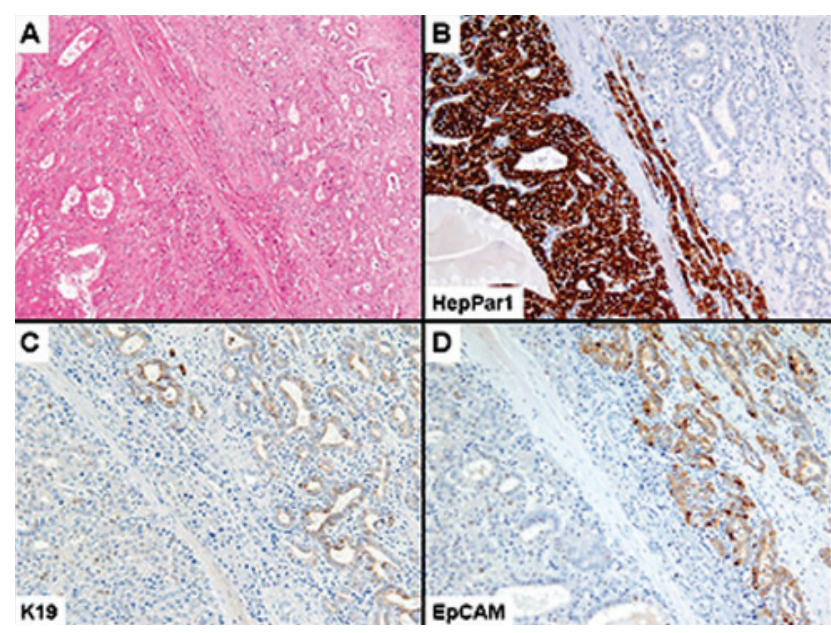

Figure 4. (A) Case 2 tumor morphology (Hematoxylin); (B) HepPar1 highlights the HCC component of the tumor; (C) Keratin 19 highlights the cholangiocarcinoma component of the tumor. (D) No stem cell features supported by a negative EpCAM stain. 
Histologically, the tumor cells are predominantly seen in pseudoacini and trabecular configurations exhibiting atypia with eosinophilic cytoplasm (Figure 4). Admixed in the surrounding desmoplastic stroma are irregular glands lined by columnar epithelium with large nuclei and prominent nucleoli. Stem cell features are not present. The tumor is seen extending to the subcapsular area and invading the vascular wall.

\section{Immunohistochemistry}

Immunostaining for EpCAM highlighted focal ductular reactions, sometimes with adjacent, clustered, EpCAM positive hepatocytes, indicative of stem/progenitor cell mediated regeneration, albeit to a small degree, of hepatic parenchyma (Figure 3C). ${ }^{11}$ Immunostains of the tumor are presented in Figure 4. The areas with hepatocellular differentiation exhibited canalicular staining for CD 10 and with polyclonal anti-CEA antibodies and strong and diffuse punctate staining for HepPar1. Stains for keratins 7 and 19 and for EpCAM were negative in these areas. Glandular components, on the other hand, stain positive for keratins 7 and 19, EpCAM and CEA (cytoplasm and membrane), but were negative for arginase-1 and HepParl.

\section{DISCUSSION}

NAFLD is the hepatic manifestation of the metabolic syndrome, a cluster of conditions that are related by obesity, insulin resistance, dyslipidemia and elevated blood pressure. ${ }^{12}$ Patients with NAFLD may show a spectrum of histologic features including steatosis, steatohepatitis and steatofibrosis, with some progressing to the complications of cirrhosis and hepatocellular carcinoma.

The clinical course of NAFLD is related to its histology at the time of diagnosis. Mild steatosis on presentation will usually connote a benign prognosis. Presence of steatohepatitis indicates an increased likelihood of disease progression that may lead to significant fibrosis. Statistically, 26\%-37\% of patients with steatohepatitis demonstrate progression to fibrosis over time, with up to $9 \%$ progressing to cirrhosis and $2 \%-5 \%$ to hepatocellular carcinoma., ${ }^{2,13-16}$ This natural history is reflected in the increasing incidence of HCC paralleling the epidemic of obesity in the United States, suggesting that NAFLD is a key factor linking obesity and HCC..$^{17-20}$

Moreover, increasing reports of HCC arising in non-cirrhotic patients with NAFLD raise the possibility that carcinogenesis occurs in NAFLD even in the absence of advanced liver disease or cirrhosis. Although uncommon, these cases show that cirrhosis is not necessary for the progression to HCC in patients with NAFLD. In this regard, data concerning contributory risk factors for its development have also become subjects of discussion. Obesity and diabetes are the two most prevalent risk factors mentioned in the literature that are associated with HCC in the background of NAFLD. ${ }^{21-22}$

Several hypotheses have been proposed in previous reports for the mechanisms of NAFLD to HCC, particularly in pre-cirrhotic stages. Malignant transformation of HCA is one suggested pathway and has been reported with a frequency of $4.2 \% .{ }^{23} \mathrm{HCA}$, though usually benign, represents a monoclonal tumor proliferation that has an inherent risk of undergoing malignant changes, more or less depending on the recently defined subtype. ${ }^{24}$ Among the different subtypes, HCA with $\beta$-catenin mutation and inflammatory HCA are the subgroups most associated with malignant progression. ${ }^{2,25}$
It is the latter form that has been presented as a precursor lesion to $\mathrm{HCC}$ in some pre-cirrhotic NAFLD cases. $^{2}$

Some investigators suggest that the prolonged exposure of hepatocytes to the toxic effects of accumulated fats is the culprit. ${ }^{7,26}$ These studies suggest that non-esterified free fatty acids (FFA) induce lipoapoptosis via the activation of the c-Jun N-terminal kinase (JNK) signaling pathway. ${ }^{27-29}$ The activation of the JNK pathways plays a pivotal role in the succeeding molecular events leading to hepatocyte apoptosis. Thus, it can be said that circulating levels of FFA correlates with liver disease severity as well as with molecular events increasing the likelihood of hepatocyte malignant transformation.

Tumor suppressor genes have also been implicated by some authors who suggest that p53, promyelocytic leukemia and PTEN genes play an important role in the development of steatosis and steatosis-induced liver cell damage. ${ }^{6-7}$ Another potential mechanism relates to dysregulated bile acid metabolism in NAFLD which has been reported to induce apoptosis and may promote HCC in the setting of hepatic steatosis. ${ }^{8}$

Our cases now suggest yet another pathway for the emergence of malignancy in non-cirrhotic NAFLD. Combined HCC-ChC (with or without stem cell features) accounts for $<1 \%$ of all liver carcinomas. ${ }^{9}$ Even without the histologic and immunophenotypic demonstration of a cell compartment with stem cell features, most combined HCC-ChC are recognized as the result of malignant transformation of a bipotent hepatobiliary stem cell. ${ }^{10}$ The finding of combined HCC-ChC with stem cell features in the background of non-cirrhotic NAFLD raises the possibility that some hepatobiliary malignancies in this disease may arise through the activation and malignant transformation of liver stem/progenitor cells in that setting.

The development of steatohepatitis and the inflammatory cascade are likely to provide the clues to the carcinogenic potential of fatty liver disease. ${ }^{1}$ The accumulation of free fatty acids potentiates the vulnerability of the liver to the byproducts of inflammation. Increase fatty acids propels the cytochrome P4502EI producing reactive oxygen species (ROS) and lipid peroxidation. ${ }^{30}$ Overproduction of these molecules depletes the anti-oxidant mechanisms causing cellular injury and oxidative stress that may lead to hepatic hyperplasia. Contributory to the inflammatory cascade and hepatocyte apoptosis is the activation of JNK1, a protein kinase also activated by ROS and free fatty acid accumulation. ${ }^{1}$ ROS together with sustained activation of JNK1 may bring about hyperplasia ${ }^{31-32}$ and increase in several genes important for hepatic proliferation. ${ }^{33}$ Furthermore, IL-6, which is markedly elevated in NAFLD, is also a potent promoter of hepatocarcinogenesis. ${ }^{34-35}$

Steatosis via the ROS and toxin production also affects the liver's inherent replicative capacity, causing its arrest leading to hepatocellular senescence. ${ }^{36}$ In effect, it thereby also stimulates hepatocellular stem/progenitor cell expansion - creating the ductular reaction - which by default becomes the source of regenerating hepatocytes. ${ }^{37}$ If the mutational events take place within the stem cell compartment directly or within their transit amplifying progeny, hepatobiliary progenitors in the ductular reaction, this pathway can lead to emergence of a combined HCC-CHC. The steatosis, thus, in some patients, may act as catalyst, through production of the above described molecules and cell/tissue reactions necessary to stimulate both stem cell activation, exposure of such cells to mutagenic events, and ultimately tumorigenesis. 


\section{CONCLUSION}

In conclusion, we present these two cases of combined hepatocellular-cholangiocarcinoma, one with overt stem cell features, as evidence for malignant transformation of hepatic stem/progenitor cells in the setting of NAFLD as one pathway of development, even when established cirrhosis is not present. These findings further underscore the increasing concern regarding the development of malignancies in non-cirrhotic NAFLD, inclusive of combined HCC-CHC in which the CHC component may indicate a particularly poor prognosis.

\section{AUTHOR DISCLOSURE}

The authors declared no conflicts of interest.

\section{REFERENCES}

1. Starley BQ, Calcagno CJ, Harrison SA. Nonalcoholic fatty liver disease and hepatocellular carcinoma: a weighty connection. Hepatology. 2010;51(5);1820-32. http://dx.doi.org/10.1002/ hep.23594.

2. Paradis V, Zalinski S, Chelbi E. et al. Hepatocellular carcinomas in patients with metabolic syndrome often develop without significant liver fibrosis: a pathological analysis. Hepatology. 2009;49(3); 851-9. http://dx.doi.org/10.1002/ hep.22734.

3. Guzman G, Brunt EM, Petrovic M, Chejfec G, Layden TJ, Cotler SJ. Does nonalcoholic fatty liver disease predispose patients to hepatocellular carcinoma in the absence of cirrhosis? Arch Pathol Lab Mab. 2008;132(11);1761-6. http://dx.doi. org/10.1043/1543-2165-132.11.1761. PMID: 18976012.

4. Bullock RE, Zaitoun AM, Aithal GP, Ryder SD, Beckingham IJ, Lobo DN. Association of non-alcoholic steatohepatitis without significant fibrosis with hepatocellular carcinoma. J Hepatol. 2004;41(4);685-6. http://dx.doi.org/10.1016/j. jhep.2004.05.008.

5. Saxena R. Practical hepatic pathology: a diagnostic approach. Philadelphia: Elsevier-Saunders, 2011.

6. Herzer K, Hofmann TG, Teufel A, et al. IFN-alphainduced apoptosis in hepatocellular carcinoma involves promyelocytic leukemia protein and TRAIL independently of p53. Cancer Res. 2009;69; 855-62. http://dx.doi. org/10.1158/0008-5472.CAN-08-2831.

7. Cazanave SC, Mott JL, Elmi NA, et al. JNK1-dependent PUMA expression contributes to hepatocyte lipoapoptosis. J Biol Chem. 2009;284; 26591-602. http://dx.doi.org/10.1074/ jbc.M109.022491.

8. Ertle J, Dechêne A, Sowa J, et al. Non-alcoholic fatty liver disease progresses to hepatocellular carcinoma in the absence of apparent cirrhosis. Int J Cancer. 2011; 128(10):2436-43. http://dx.doi.org/10.1002/ijc.25797.

9. Theise ND, Nakashima O, Park YN, Nakanuma Y. Combined hepatocellular-cholangicarcinoma. In: Bosman FT, Carneiro F, Hruban RH, Theise ND, eds. WHO Classification of tumours of the digestive system. Lyon: IARC;2010, pp. 225-7.

10. Theise ND, Yao JL, Harada K, et al. Hepatic "stem cell" malignancies in adults: four cases. Histopathology. 2003;43(3);263-71. http://dx.doi.org/10.1046/j.1365-2559.2003. 01707.x. PMID: 12940779.

11. Yoon SM, Gerasimidou D, Kuwahara R, et al. Epithelial cell adhesion molecule (EpCAM) marks hepatocytes newly derived from stem/progenitor cells in humans. Hepatology. 2011;53(3);964-73. http://dx.doi.org/10.1002/hep.24122.
12. Brunt EM. Nonalcoholic steatohepatitis. Semin Liver Dis.2004;24(1);3-20.http://dx.doi.org/10.1055/s-2004-823098.

13. Harrison SA, Torgerson S, Hayashi PH. The natural history of nonalcoholic fatty liver disease: a clinical histopathological study. Am J Gastroenterol. 2003;98;2042-7. http://dx.doi. org/10.1111/j.1572-0241.2003.07659.x.

14. Adams LA, Sanderson S, Limdor KD, Angulo P. The histological course of nonalcoholic fatty liver disease: a longitudinal study of 103 patients with sequential liver biopsies. J Hepatol .2005;42(1);132-8. http://dx.doi.org/10.1016/j. jhep.2004.09.012.

15. Fassio E, Álvarez E, Domínguez N, Landeira G, Longo C. Natural history of nonalcoholic steatohepatitis: a longitudinal study of repeat liver biopsies. Hepatology. 2004;40(4);820-6. http://dx.doi.org/10.1002/hep.1840400411.

16. Lindor KD, Kowdley KV, Heathcote EJ, Harrison ME, Jorgensen R, Angulo P, et al. Ursodeoxycholic acid for treatment of nonalcoholic steatohepatitis: results of a randomized trial. Hepatology. 2004;39(3);770-8. http://dx.doi. org/10.1002/hep.20092.

17. Caldwell Sh, Oelsner DH, Iezzoni JC, Hespenheide EE, Battle EH, Driscoll CJ. Cryptogenic cirrhosis: clinical characterization and risk factors for underlying disease. Hepatology. 1999;29(3);664-9. http://dx.doi.org/10.1002/ hep.510290347.

18. Bugianesi E, Leone N, Vann E, et al. Expanding the natural history of nonalcoholic steatiohepatitis: from cryptogenic cirrhosis to hepatocellular carcinoma. Gastroenterology. 2002;123(1);134-140. http://dx.doi.org/10.1053/gast.2002. 34168.

19. Hai S, Kubo S, Shuto T, et al. Hepatocellular carcinoma arising from nonalcoholic steatohepatitis: report of two cases. Surg Today. 2006;36(4);390-4. http://dx.doi.org/10.1007/ s00595-005-3167-4.

20. Mori S, Yamasaki T, Sakaida I et al. Hepatocellular carcinoma with nonalcoholic steatohepatitis. J Gastroenterol. 2004;39(4);391-6. http://dx.doi.org/10.1007/s00535-0031308-3.

21. Cuadrado A, Orive A, Garcia-Suárez C, et al. Nonalcoholic steatohepatitis (NASH) and hepatocellular carcinoma. Obes Surg. 2005;15(3);442-6. http://dx.doi. org $/ 10.1381 / 0960892053576596$.

22. Regimbeau JM, Colombat M, Mognol P, et al. Obesity and diabetes as a risk factor for hepatocellular carcinoma. Liver Transpl. 2004;10(Suppl 2);S69-73. http://dx.doi.org/10.1002/ lt. 20033.

23. Stoot JHMB, Coelen RJS, De Jong MC, Dejong CHC. Malignant transformation of hepatocellular adenomas into hepatocellular carcinomas: a systematic review including more than 1600 adenoma cases. HPB. 2010;12(8);509-22. http://dx.doi.org/10.1111/j.1477-2574.2010.00222.x.

24. Paradis V, Laurent A, Flejou JF, Vidaud M, Bedossa P. Evidence for the polyclonal nature of focal nodular hyperplasia of the liver by the study of X-chromosome inactivation. Hepatology. 1997;26(4);891-5. http://dx.doi.org/10.1002/ hep.510260414.

25. Farges O, Dokmak S. Malignant transformation of liver adenoma: an analysis of the literature. Dig Surg. 2010;27(1);32-8. http://dx.doi.org/10.1159/000268405.

26. Unger RH, Orci L. Lipoapoptosis: its mechanism and its diseases. BBA-Mol Cell Biol L. 2002;1585(2-3); 202-12.

27. Puri P, Mirshahi F, Cheung O, et al. Gastroenterology. 2008;134(2):568-76. http://dx.doi.org/10.1053/j.gastro.2007. 10.039 . 
28. Singh R, Wang Y, Xiang Y, Tanaka KE, Gaarde WA, Czaja MJ. Hepatology. 2009; 49(1);87-96. http://dx.doi.org/10.1002/ hep. 22578 .

29. Schattenberg JM, Singh R, Wang Y, et al. JNK1 but not JNK2 promotes the development of steatohepatitis in mice. Hepatology. 2006;43:163-72.

30. Clouston A, Powell E. Interaction of non-alcoholic fatty liver disease with other liver diseases. Best Pract Res Clin Gastroenterol. 2002;16(5);767-81. http://dx.doi.org/10.1053/ bega.2002.0329.

31. Bugianesi E. Non-alcoholic steatohepatitis and cancer. Clin Liver Dis. 2007;11(1):191-207,x-xi. PMID: 17544979.

32. Yang S, Zhu H, Li Y, Lin H, Gabrielson K, Trush MA, et al. Mitochondrial adaptations to obesity-related oxidant stress. Arch Biochem Biophys. 2000;378(2);259-68. http://dx.doi. org/10.1006/abbi.2000.1829.

33. Chen F, Beezhold k, Castranova V. JNK1, a potential therapeutic target for hepatocellular carcinoma. BBA-Rev Cancer. 2009;1796(2);242-51. http://dx.doi.org/10.1016/j. bbcan.2009.06.005.
34. Park EJ, Lee JH, Yu GY, et al. Dietary and genetic obesity promote liver inflammation and tumorigenesis by enhancing IL-6 and TNF expression. Cell. 2010;140(2);197-208. http:// dx.doi.org/10.1016/j.cell.2009.12.052.

35. Hatziapostolou M, Polytarchou C, Aggelidou E, et al. An HNF4a-miRNA inflammatory feedback circuit regulates hepatocellular oncogenesis. Cell. 2011;147(6);1233-47. http://dx.doi.org/10.1016/j.cell.2011.10.043.

36. Clouston AD, Powell EE, Walsh MJ, Richardson MM, Demetris AJ, Jonsson JR. Fibrosis correlates with a ductular reaction in hepatitis C: roles of impaired replication, progenitor cells and steatosis. Hepatology. 2005;41(4);809-18. http://dx.doi.org/10.1002/hep.20650.

37. Sell S. Heterogeneity and plasticity of hepatocyte lineage cells. Hepatology. 2001;33(3);738-50. http://dx.doi.org/10.1053/ jhep.2001.21900.

Disclaimer: This journal is OPEN ACCESS, providing immediate access to its content on the principle that making research freely available to the public supports a greater global exchange of knowledge. As a requirement for submission to the PJP, all authors have accomplished an AUTHOR FORM, which declares that the ICMJE criteria for authorship have been met by each author listed, that the article represents original material, has not been published, accepted for publication in other journals, or concurrently submitted to other journals, and that all funding and conflicts of interest have been declared. Consent forms have been secured for the publication of information about patients or cases; otherwise, authors have declared that all means have been exhausted for securing consent. 